\title{
A short introduction to shadows of 4-manifolds
}

\author{
by \\ Francesco Costantino (Strasbourg)
}

\begin{abstract}
We give a self-contained introduction to the theory of shadows as a tool to study smooth 3-manifolds and 4-manifolds. The goal of the present paper is twofold: on the one hand, it is intended to be a shortcut to a basic use of the theory of shadows, on the other hand it gives a sketchy overview of some of the most recent results on shadows. No original result is proved here and most of the details of the proofs are left out.
\end{abstract}

1. Introduction. Shadows were defined by V. Turaev for the first time at the beginning of the nineties in [19] as a method for representing knots alternative to the standard one based on knot diagrams and Reidemeister moves. The theory was then developed in the preprint "Topology of shadows" which was later included in revised form in [17]; moreover, a short account of the theory was published by Turaev in [18]. Since then, probably due to the slightly higher degree of complication of this theory with respect to Kirby calculus, only a few applications of shadows have been studied. Among these, we recall the use of shadows to study Jones-Vassiliev invariants of knots by U. Burri in [3] and A. Shumakovitch in [15] and the study of "interdependent modifications of links and invariants of finite degree" by M. N. Goussarov in [10].

In the last two years, there has been a new surge of interest in shadows testified by the appearance of the preprint [16] of D. Thurston on knotted trivalent graphs and shadows and by the study of a new notion of complexity of 3-manifolds based on shadows developed by the author and D. Thurston in [9]. This notion of "shadow complexity" turns out to be intimately connected with the geometric properties of 3-manifolds and in particular with their hyperbolic structures; this fact was also used in [7] to exhibit and study a particular class of 3-manifolds having strong geometric properties. Moreover a refinement of the notion of shadow of a 4-manifold, called a "branched

2000 Mathematics Subject Classification: 52B70, 57N13.

Key words and phrases: polyhedra, manifolds, shadow, spine. 
shadow", has been studied by the author in [6], to attack a combinatorial study of gauge invariants and complex convexity problems. Branched shadows allow one to encode homotopy classes of almost complex structures on 4-manifolds, which, on manifolds admitting a shadow, are in a natural bijection with the set of $\mathrm{Spin}^{c}$-structures. Moreover, it has been proved by the author that branched shadows embedded in complex manifolds behave like real surfaces; in particular a classification of the points of a branched shadow into totally real, complex elliptic and complex hyperbolic ones is possible. These arguments have then been used to study geometric problems as the existence of integrable representatives of homotopy classes of almost complex structures or the existence of Stein domain structures on a neighborhood of a shadow.

Roughly speaking, shadows of 4-manifolds are 2-dimensional polyhedra embedded in 4-manifolds equipped with extra structures suitable to encode the topology of their regular neighborhood; they represent the analogs of spines of 3-manifolds in the 4-dimensional case. Shadows allow a completely combinatorial approach similar to the one used in the 3-dimensional case by means of spines.

Since the first obstacle to the comprehension of shadows is the familiarity with simple polyhedra, in Section 2 we clarify what a simple polyhedron is and how it can be combinatorially constructed by means of a finite set of basic building blocks. Sections 3-5 are devoted to the basic facts of the theory of shadows proved by Turaev in [17]. In Section 3, we study how an embedding of a polyhedron into a 4-manifold can be used to equip the polyhedron with an additional structure, called "gleam" by Turaev, which is fundamental in the theory. In Section 4, we recall Turaev's method to thicken a polyhedron equipped with gleams and to construct 4-manifolds. In Section 5, we introduce a set of basic moves on shadows and study their effect on the corresponding 4-manifolds.

The last three sections are devoted to an overview of some of the open problems and questions. In Section 6, we outline connections between shadows of 4-manifolds and the Andrews-Curtis conjecture. Section 7 is devoted to the results proven recently by the author and D. Thurston on shadow complexity and hyperbolic geometry of 3-manifolds. Finally, in Section 8 we outline some of the recent developments of the theory towards a combinatorial method for studying 4-manifolds equipped with additional structures such as $\operatorname{Spin}^{c}$, almost-complex, complex and Stein structures.

Acknowledgements. The author wishes to warmly thank Riccardo Benedetti, Dylan Thurston and Vladimir Turaev for their criticism and encouraging comments. 
2. Simple polyhedra. The basic objects underlying shadows of 4manifolds are simple, 2-dimensional polyhedra, that is, 2-dimensional CWcomplexes whose points have regular neighborhoods of "simple" type as those shown in Figure 1. From the figure, we observe that the set of points

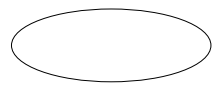

Region

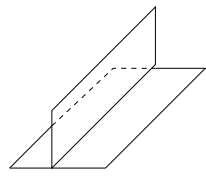

Edge

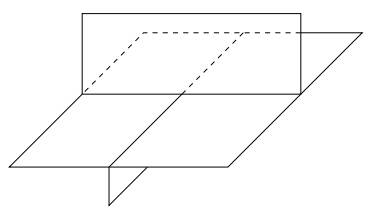

Vertex

Fig. 1. The local models of a simple polyhedron

whose regular neighborhood in a simple polyhedron $P$ is shaped like the last two models, and which is called the singular set of the polyhedron, is a 4-valent graph denoted by $\operatorname{Sing}(P)$ whose vertices are exactly the points whose regular neighborhoods are shaped like the third model and are called vertices of the polyhedron. The complement of the singular set of the polyhedron is the union of a set of open surfaces embedded in the polyhedron and called regions.

One can check that the local pattern around a vertex is homeomorphic to the regular neighborhood of the vertex of the cone over the edges of a tetrahedron; this shows that the full symmetry group of the regular neighborhood of a vertex is the group of permutations of four elements. Another way of visualizing a vertex is to consider the result of attaching the polyhedron $Y \times[0,1]$ (where $Y$ is a $Y$-shaped graph) along $Y \times 0$ to a $Y$-shaped graph embedded in a disc.

To clarify the reason why we restrict only to the three kinds of local singularities of Figure 1 we shift to the one-dimensional case where the analog of simple polyhedra is the set of graphs whose local possible patterns are the one homeomorphic to the open interval and the one homeomorphic to an open $Y$-shaped graph; in this case the vertices are the vertices of the graph. A generic graph could contain some $n$-valent vertex (with $n>3$ ), so that the restriction to 3 -valent graphs appears somewhat strong. Roughly speaking, the idea underlying this choice is that an $n$-valent vertex of a graph is "stable" only if $n=3$ : if we slightly perturb the position of an edge of the graph near, say, a 4 -valent vertex, we can transform the vertex by a local modification into a pair of 3 -valent vertices, passing from an $X$-shaped graph to an $H$-shaped one; if we try to repeat this operation for one of the two 3-valent vertices obtained that way, we cannot further modify the homeomorphism type of the graph. More formally (but we will not go into these matters), each graph is simply-homotopic to a trivalent 
one. Analogously, in dimension 2, each polyhedron can be suitably perturbed to a new one whose singularities are those depicted in Figure 1.

The first example of simple polyhedron is a closed surface, whose points are all of the first type in Figure 1. An example containing only points of the first two types is the union of a sphere in $\mathbb{R}^{3}$ and the disc spanned by a maximal circle in it and contained in the ball bounded by the sphere. This polyhedron has singular set forming a circle and contains no vertices; its regions are three (open) discs.

A third example can be constructed by using the last one and gluing to it along another equator (different from the one already chosen) another disc, this time contained in the complement of the ball bounded by the initial sphere. The polyhedron we get has a singular set containing two 4-valent vertices (the intersections of the two maximal circles in the sphere used to construct it) and such that no edge of the graph has endpoints in the same vertex; the regions of the polyhedron are six discs.

In the above example we used a sphere embedded in $\mathbb{R}^{3}$ but this was only for the sake of clarity: one of the main properties of simple polyhedra is that they can be abstractly described by means of their combinatorial structure. To give an idea of how this can be performed we do this for the preceding example.

First, one identifies the structure of the singular set: in our case a 4valent graph with two vertices connected by four edges. Then one studies how each region is glued to the singular graph: in our case the boundary of each region passes exactly once over two different edges of the singular set and zero times over the others. Moreover, for each pair of edges there is exactly one region whose boundary is the union of the two edges: there are four edges and hence six pairs of edges and exactly six regions. Hence, to reconstruct the polyhedron, it is sufficient to start from the 4-valent graph and glue the six regions over all the possible pairs of edges.

In the preceding case, we were treating a particular type of polyhedron: all its regions were discs and its singular graph was connected; such polyhedra are called standard. The above combinatorial reconstruction is applicable also in the general case of simple polyhedra, but it is easier to describe in the standard case since there is no need of specifying the topology of each region to be glued to the singular graph. From now on, for brevity, we will use the word polyhedron for standard polyhedron, restricting the set of objects we will study; anyway, all our discussions will also hold for simple polyhedra in general.

Before ending this section, we define the notion of "mod 2 gleam" of a polyhedron $P$. Let $D$ be a region of $P$, and consider the regular neighborhood of $\partial D$ in $P$. For simplicity, we suppose that $D$ is embedded in $P$, but the same arguments apply to the general case when $\partial D$ runs more than once 
over an edge of $\operatorname{Sing}(P)$. The regular neighborhood of a point $p_{0}$ of $\partial D$ different from a vertex of $P$ is formed by a half plane (corresponding to $D$ ) glued along a segment to a square in $\mathbb{R}^{2}$ which is split in two components, corresponding to the other regions touching $D$ along its boundary near $p_{0}$. Choose one of these components, and continue this choice along $\partial D$ until we reach a vertex of $P$. To extend this choice near the vertex, we delete from the neighborhood of the vertex the only region which locally touches $D$ only in the vertex itself (there are six regions around a vertex and they are "opposite" in pairs): we obtain again a square divided in two by $\partial D$ and we can continue our choice canonically (we recommend the reader to try to visualize it). After completing a whole loop around $\partial D$, we come back to the initial point $p_{0}$ with a choice of one of the two components into which $\partial D$ splits the neighborhood of $p_{0}$ in $P-D$. If this second component is the one we chose initially then we say that the $\bmod 2$ gleam of $D$ is 0 , otherwise it is 1 . Acting similarly for each region of $P$ we get a $\mathbb{Z}_{2}$-coloring on the regions of $P$ called $\bmod 2$ gleam of $P$.

In the case of an embedded region $D$, the above description can be summarized as follows: consider the regular neighborhood of $\partial D$ in $P-\operatorname{int}(D)$; if it collapses over a Möbius strip, then the mod 2 gleam of $D$ is 1 , otherwise it is 0 .

3. Polyhedra in 4-manifolds. In this section we investigate how a polyhedron $P$ embedded in a 4-manifold $M$ can be given an extra structure related to the topology of its regular neighborhood. Let us be more specific regarding the word "embedded":

Definition 3.1. A polyhedron $P$ embedded in a smooth 4-manifold $M$ is said to be locally flat if for each point $p$ of $P$ there is a local chart $(U, \phi)$ of the smooth atlas of $M$ such that the image of $P \cap U$ under $\phi$ is contained in a 3-dimensional plane in which it appears as one of the three local pictures of Figure 1 in $\mathbb{R}^{3} \subset \mathbb{R}^{4}$, that is, around each of its points, $P$ is contained in a 3-dimensional slice of $M$ and in this slice it appears as shown in Figure 1.

For brevity, from now on we will use the word "embedded" for "locally flat embedded". The first question we ask is the following: can we reconstruct the regular neighborhood of a polyhedron $P$ in a manifold $M$ from the combinatorics of $P$ ? Before considering the 4-dimensional case, let us understand the easier 3 -dimensional case, where $P$ is a polyhedron embedded in an oriented 3 -manifold $N$. In this case one sees that the $\mathbb{Z}_{2}$-gleam of each region of $P$ is 0 : indeed, roughly speaking, the regular neighborhood of a region of $P$ (which is a disc) in $N$ is $D^{2} \times[-1,1]$ and so it is disconnected by the region; this would be false if the mod 2 gleam of the 

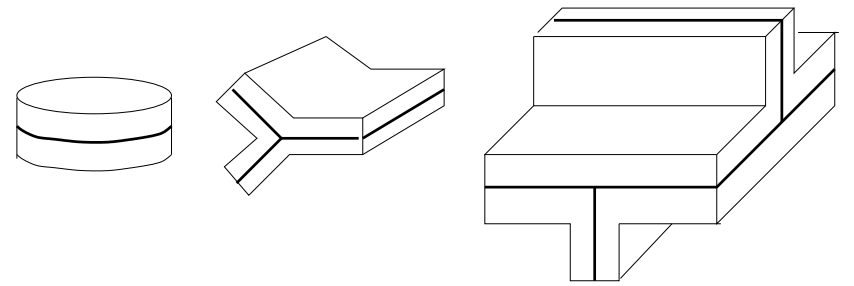

Fig. 2. The three types of blocks used to thicken a spine of a 3-manifold

region were 1 . Hence, the fact that a polyhedron embeds in a 3-manifold imposes some restrictions on its combinatorics. The truth is much deeper: the combinatorics of the polyhedron allows one to reconstruct the topology of its regular neighborhood in $N$. Indeed, by decomposing $P$ into the local patterns shown in Figure 1, we can decompose its regular neighborhood in blocks as in Figure 2 which can be glued back to each other according to the combinatorics of $P$. That way, a polyhedron with zero mod 2 gleam determines a 3-manifold collapsing onto it. It can be shown that any 3-manifold with non-empty boundary can be constructed that way, and so the study of 3 -manifolds can be attacked by means of particular polyhedra; this is the basic idea underlying the theory of spines of 3-manifolds.

Let us now turn to the 4-dimensional case and, for simplicity, choose $P$ to be homeomorphic to $S^{2}$. Suppose that $P$ is embedded in a smooth, oriented 4-manifold $M$; is it possible to reconstruct its regular neighborhood using only its topology? The answer is "no" since the regular neighborhood of a sphere (and more generally of a surface) is determined by the topology of the surface and by its self-intersection number in the manifold. To state it differently, the regular neighborhood of a surface in a 4-manifold is diffeomorphic to the total space of a disc bundle over the surface (its normal bundle) and the Euler number of this bundle is a necessary datum to reconstruct its topology.

Hence, we see that to codify the topology of the regular neighborhood of $P$ in $M$ we need to decorate $P$ with some additional information; when $P$ is a surface, the Euler number of its normal bundle is a sufficient datum.

More generally the following holds:

Proposition 3.2. Let $P$ be a polyhedron embedded in an oriented smooth 4-manifold $M$. There is a coloring of the regions of $P$ with values in the half integers $\frac{1}{2} \mathbb{Z}$ canonically induced by its embedding in $M$. This coloring is called the gleam of $P$ in $M$.

Proof. The construction we will describe is similar to the one used to define the mod 2 gleam of a polyhedron. For simplicity let again $D$ be a region of $P$ whose boundary is embedded in $\operatorname{Sing}(P)$. Let $p_{0}$ be a point of $\partial D$ and consider the 3 -dimensional slice $B^{3}$ of $M$ in which a regular neighborhood 
of $p_{0}$ in $P$ is sitting (it exists since by hypothesis $P$ is locally flat in $M$ ). Fix an auxiliary riemannian metric on $M$ and consider the direction orthogonal to $D$ in $B^{3}$; by construction, it coincides with the direction along which the two other regions touching $\partial D$ on $p_{0}$ get separated (see Figure 3 ).

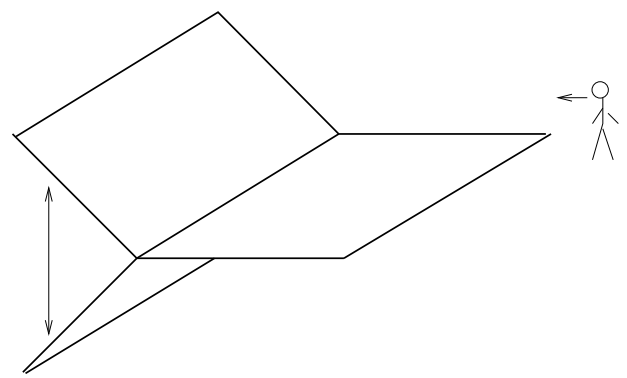

Fig. 3. The picture sketches the position of the polyhedron in a 3-dimensional slice of the ambient 4-manifold. The direction indicated by the vertical double arrow is the one along which the two regions touching the horizontal one get separated.

We can extend the definition of this direction to the whole $\partial D$, in the same way we did to get the mod 2 gleam, obtaining a continuous choice of directions orthogonal to $D$ in $M$ along $\partial D$. This choice represents a section of the bundle of directions orthogonal to $D$ in $M$, whose fiber is $S^{1}$. The obstruction to extend this section to the whole $D$ can be canonically identified with an integer: indeed, it represents an element of $H^{2}\left(D, \partial D ; \pi_{1}\left(S^{1}\right)\right)$ and if we fix an orientation on $D$ then also the fiber gets oriented since $M$ is oriented, thus giving an identification of $H^{2}\left(D, \partial D ; \pi_{1}\left(S^{1}\right)\right)$ with $H^{2}(D, \partial D ; \mathbb{Z})$ $=\mathbb{Z}$; this identification does not change if we revert the orientation of $D$, since also the orientation of the fiber changes. Hence, if we divide by 2 the number obtained through this identification, we get an element of $\frac{1}{2} \mathbb{Z}$ which we will call the gleam of $D$, and which represents the Euler number of the normal bundle of $D$ in $M$. It is worth noting that the Euler number of the normal bundle of an embedded surface equals the self-intersection number of the surface, so that the gleam represents a version of the self-intersection number "localized" on the regions of the polyhedron.

The above construction can be performed analogously in the case when $D$ is not embedded in $P$; that way, we can define a gleam over each region of $P$. Note that, by construction, the gleam of a region of $P$ is non-integral (an odd multiple of $1 / 2$ ) if and only if its mod 2 gleam is 1 .

4. From polyhedra with gleams to 4-manifolds. In the preceding section we showed that any polyhedron embedded in a 4-manifold can be equipped with a gleam. This produces a map from the pairs $(M, P)$ of smooth, oriented and compact manifolds containing embedded polyhedra 
into the set of polyhedra equipped with gleams, $(P, \mathrm{gl})$, where a gleam is a $\frac{1}{2} \mathbb{Z}$ coloring of the regions of $P$ such that the color of a region is non-integral if and only if its mod 2 gleam is 1 . We also stated that if $P$ is a surface then its gleam is the Euler number $e$ of the normal bundle of the surface in $M$ and hence the pair $(P, e)$ is sufficient to reconstruct the regular neighborhood of $P$ in $M$. This is true in general as proved by Turaev in [17]:

Theorem 4.1 (Reconstruction map). Let $(P, \mathrm{gl})$ be a polyhedron equipped with gleams. There exists a canonical reconstruction map associating to $(P, \mathrm{gl})$ a pair $\left(M_{P}, P\right)$ where $M_{P}$ is a smooth, compact and oriented 4manifold containing an embedded copy of $P$ over which it collapses and such that the gleam of $P$ in $M_{P}$ coincides with the initial gleam gl. The pair $\left(M_{P}, P\right)$ can be explicitly reconstructed from the combinatorics of $P$ and from gl. Moreover, if $P$ is a polyhedron embedded in a smooth and oriented manifold $M$ and $\mathrm{gl}$ is the gleam induced on $P$ as explained in the preceding section, then $M_{P}$ is diffeomorphic to a neighborhood of $P$ in $M$.

The above theorem is the key tool of the theory of shadows of 4-manifolds: indeed, by means of this result, one can study 4-manifolds in a purely combinatorial way, using only polyhedra equipped with gleams.

Proof of 4.1. The idea of the proof is to decompose $P$ into blocks as those of Figure 1, thicken them to canonical blocks, and then describe how to glue these blocks according to the combinatorics of $P$ and to its gleam. The shape of the basic blocks is easily understood: consider the blocks used to reconstruct 3-manifolds from their spines and take their products with $[-1,1]$. Then the block corresponding to a region is $D^{2} \times D^{2}$ and the blocks corresponding to edges and vertices of $\operatorname{Sing}(P)$ are the products respectively of the second and third block of Figure 2 with a segment $[-1,1]$ representing the "fourth dimension".

We first reconstruct the regular neighborhood in $M_{P}$ of the subpolyhedron $S$ of $P$ coinciding with the regular neighborhood of $\operatorname{Sing}(P)$ in $P$; to do this, we use the blocks corresponding to vertices and edges of $P$. The combinatorics of $P$ forces us to glue these blocks to each other and leaves us only one choice: gluing them by reverting or not the $[-1,1]$ factor of the fourth dimension of the blocks. If $P$ contains $n$ vertices, we have to connect the $n$ blocks corresponding to these vertices through the $2 n$ blocks corresponding to edges of the 4-valent graph $\operatorname{Sing}(P)$. Consider a maximal subtree $T$ of $\operatorname{Sing}(P)$, and glue the blocks corresponding to the vertices and edges of $T$ according to the combinatorics of $S$ choosing arbitrarily the gluing on the level of the $[-1,1]$ factors, that is, choosing arbitrarily whether to identify the $[-1,1]$ fibers by the identity or the multiplication by -1 . The manifold constructed that way is $B^{4}$ and contains a properly embedded copy of a regular neighborhood of $T$ in $S$. Only $n+1$ blocks corresponding to the edges 
of $\operatorname{Sing}(P)-T$ are now left to be glued according to the combinatorics of $S$. For each of these blocks, there is only one way of gluing the $[-1,1]$ fiber to get an orientable 4-manifold; moreover, at the end of this construction, the manifold $H$ obtained is diffeomorphic to the regular neighborhood of a 4-valent graph $(\operatorname{Sing}(P))$ in $\mathbb{R}^{4}$.

Note that since $H$ admits an orientation reversing diffeomorphism corresponding to the product by -1 along the fiber $[-1,1]$ of the blocks we used to construct $H$, it is canonically oriented. Moreover, by construction, $H$ fibers over a (not necessarily orientable) 3 -manifold $L$ whose spine is $S$; the fiber is exactly the $[-1,1]$ factor of the blocks used to construct $H$. To construct $L$ it is sufficient to repeat the above construction of $H$ using the 3 -dimensional blocks of Figure 2 without taking the product with $[-1,1] ; L$ is not necessarily orientable, and since its construction does not deal with the regions of $P$, no assumption on the gleams of the regions of $P$ is necessary to construct $L$ or $H$. Finally, a copy of $S$ is properly embedded in $L$; then it is embedded in a locally flat way in $H$ so that its boundary is contained in $\partial H$. Each boundary component of $S$ corresponds by construction to the boundary component of a region of $P$, hence to finish our construction we have to glue along $\partial S \subset \partial H$ the blocks corresponding to the regions of $P$.

Let us show how to do this for a single region $Y$ of $P$. The block corresponding to $Y$ is $Y \times D^{2}$, and since by hypothesis $Y$ is a disc, it is a 4-ball with a prescribed 2-handle structure, $Y$ being the core of the handle. To describe how to glue this 2-handle to $H$, we:

(1) identify the image of $\partial Y$ in $\partial H$ under the gluing map;

(2) identify the image in $\partial H$ under the gluing map of the canonical framing of $\partial Y$, that is, of the curve $\partial Y \times\{1\}$.

The first step is straightforward: $\partial Y$ has to be identified with the boundary component $s$ of $S$ contained in $\partial H$ corresponding to $Y$. To perform the second step, we have to identify a canonical framing on $s$; this can be done since $H$ fibers over $L$ with fiber $[-1,1]$. Indeed, while assembling $H$, we glued blocks having the structure of products of 3-dimensional blocks and $[-1,1]$ and we always respected this product structure up to the orientation of the $[-1,1]$ fiber. Hence each boundary component of $S \subset H$ (and in particular $s$ ) is automatically equipped with the framing given by the $[-1,1]$ direction; the only delicate point is that the fiber over $s$ of the projection of $H$ onto the $L$ could in some cases be a Möbius strip instead of an annulus. If it is an annulus, we have a genuine framing on $s$ and hence, to attach the 2-handle corresponding to $Y$, it is sufficient to say how many times the image of the canonical framing of $\partial Y$ in $Y \times D^{2}$ twists around $s$ in $\partial H$ with respect to this framing; since $H$ is oriented, an integer is then sufficient to encode this information, and we use the gleam of $Y$. Indeed, the gleam of $Y$ 
is an integer exactly when the inverse image in $H$ of the curve $s$ under the projection along the $[-1,1]$ fiber is an annulus.

We are left with the case when the inverse image of $s$ in $H$ under the projection along the $[-1,1]$ direction is a Möbius strip $M_{s}$. In this case, the gleam of $Y$ is a half integer; we then add a positive half twist to $M_{s}$ around $s$ (recall that $H$ is oriented), obtaining an annulus whose core is $s$ and hence a framing on $s$. We now perform the construction of the preceding case using as new gleam for $Y$ the integer given by the initial gleam of $Y$ minus 1/2.

Performing the above construction for all the regions of $P$ we get a 4dimensional "neighborhood" of $P$; it can be checked that the gleam induced on $P$ by this neighborhood coincides with the initial gleam gl.

EXAmple 4.2. Let $P$ be a spine of an orientable 3 -manifold $N$; in particular its mod 2 gleam has to be zero. By performing the construction above, using as gleam on $P$ the 0 -gleam over all the regions, we get the manifold $N \times[-1,1]$.

REMARK 4.3. All the 4-manifolds obtained by "thickening" the polyhedra equipped with gleams as in the proof of Theorem 4.1 admit a handle decomposition containing no handles of index greater than 2 . It can be shown that also the converse holds: any manifold admitting such a handle decomposition can be obtained by applying Theorem 4.1 to a suitable polyhedron equipped with gleams (see [4]) .

Definition 4.4. A polyhedron $(P, \mathrm{gl})$ equipped with gleams is a shadow of a 4-manifold $M$ if $M$ is diffeomorphic to the thickening $M_{P}$ of $P$ obtained through Theorem 4.1.

The above remark shows that shadows can be used to describe combinatorially only a subset of all the smooth 4-manifolds not including closed ones. To obviate this apparent weakness of the theory, let us recall the following result due to F. Laudenbach and V. Poenaru [12]:

THEOREM 4.5. Let $M$ be an oriented, smooth and compact 4-manifold with boundary equal to $S^{3}$ or to a connected sum of copies of $S^{2} \times S^{1}$. Then there is only one (up to diffeomorphism) closed, smooth and oriented 4-manifold $M^{\prime}$ obtained by attaching to $M$ only 3-handles and 4-handles.

Roughly speaking, the above result states that when a manifold is "closable" then it is so in a unique way (up to diffeomorphism). This allows us to describe all the closed 4-manifolds by means of polyhedra with gleams: indeed, given a closed manifold equipped with an arbitrary handle decomposition, considering the union of all handles of index strictly less than 3 we get a new manifold $M$ which admits a shadow and can be described combinatorially as explained above. The initial manifold can then be uniquely recovered from $M$ because of Theorem 4.5. We can then give the following definition: 
Definition 4.6. A polyhedron with gleams $(P, \mathrm{gl})$ is a tunnel shadow of a closed 4-manifold $X$ if $X$ can be obtained by attaching 3-handles and 4-handles to the 4-manifold $M_{P}$ obtained from $P$ through the reconstruction map of Theorem 4.1.

The name "tunnel shadow" comes from the fact that the inverse of attaching a 3-handle is the operation of digging a tunnel along a properly embedded arc. We will see later how to construct a shadow of the complement of a properly embedded arc in the thickening $M_{P}$ of a shadow $P$.

EXAmple 4.7. Let $P$ be equal to a 2 -sphere and let its gleam be 1 . The thickening of $(P, \mathrm{gl})$ is the total space of the disc bundle over $S^{2}$ with Euler number 1 , whose boundary is $S^{3}$. This space is the complement of a point in $\mathbb{C P}^{2}$, and, by Theorem $4.5,(P, \mathrm{gl})$ is a tunnel shadow of $\mathbb{C P}^{2}$.

5. Shadows and basic moves. In this section we outline the list of basic modifications of polyhedra with gleams called moves which allow one to produce new shadows of the same manifold from a given one.

From now on, let $(P, \mathrm{gl})$ be a shadow of a 4 -manifold $M$ into which it is embedded through the construction of the proof of Theorem 4.1. The first three moves we describe are called shadow equivalences; they modify the position of $P$ in $M$ producing a new shadow $P^{\prime}$ embedded in $M$ which differs from $P$ only inside a 4-ball.

These moves are shown in Figure 4; for the moment let us forget about the letters written in the figure. To visualize these moves, imagine that a region of $P$ is slid over some other regions, producing a polyhedron which is different from the initial one only in a contractible subpolyhedron (the one pictured in the left part of the figure). These moves are called respectively $1 \rightarrow 2,0 \rightarrow 2$ and $2 \rightarrow 3$ moves, because of their effect on the number of vertices of the polyhedra. Since the $1 \rightarrow 2$ move is the trickiest to understand, we concentrate first on the $0 \rightarrow 2$ move (also called finger move or lune move); this move acts in a 4-ball contained in $M$ and containing the part of $P$ shown in the left part of the picture. After the move, we replace this part of $P$ with the new one drawn in the right part of the figure and we keep the 4-ball unchanged, obtaining a shadow $P^{\prime}$ of the same 4-manifold $M$. One can imagine the move as a sliding over the horizontal regions of the region which in the left part of the figure is the upper-left; this move can be performed in a 3 -dimensional slice of the 4 -ball in $M$; hence we say that the $0 \rightarrow 2$ move has a 3 -dimensional nature. The same holds for the $2 \rightarrow 3$ move, also called the Matveev-Piergallini move: although apparently more complicated than the $0 \rightarrow 2$ move, it can be seen that it is a version of the $0 \rightarrow 2$ move in which the sliding region passes over a vertex. The same comments as above apply to this case. 


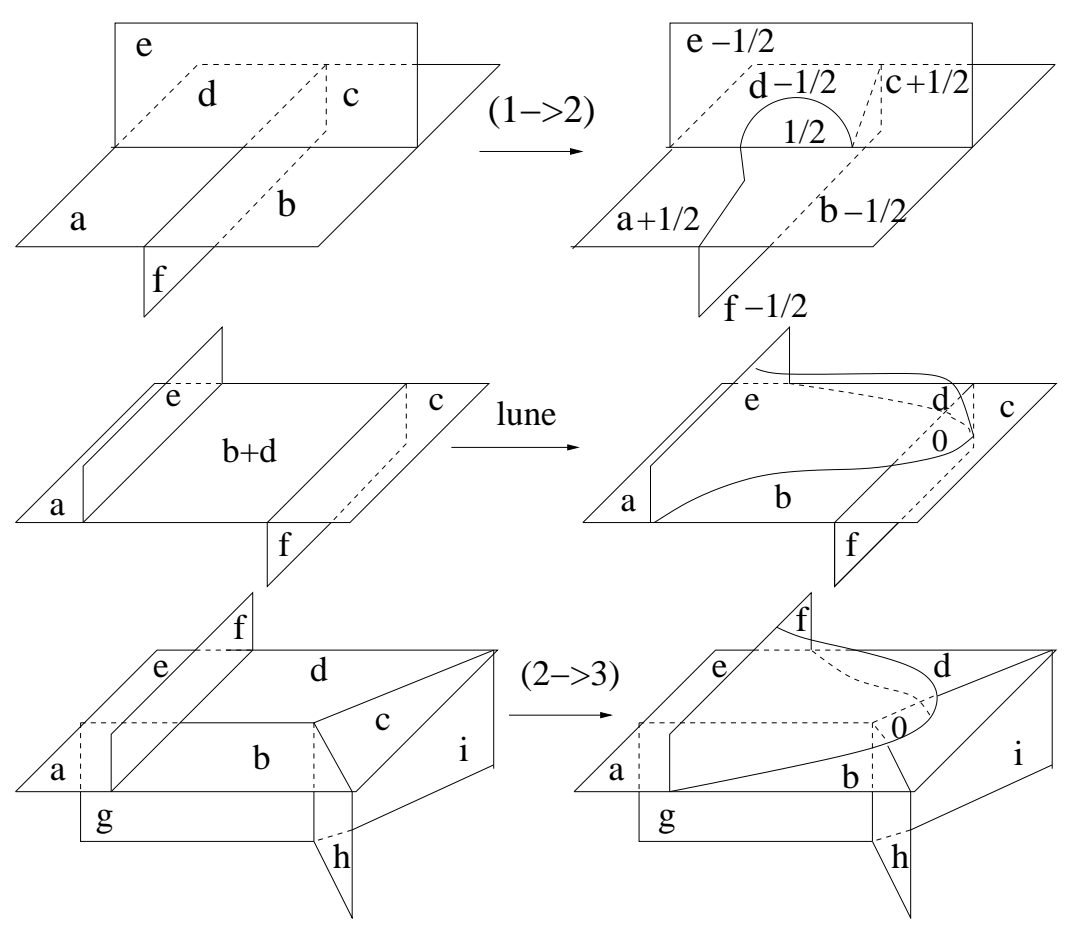

Fig. 4. The three shadow equivalences

The case of the $1 \rightarrow 2$ move is different; this move applies to a neighborhood of a vertex and lets a region (the vertical lower one in the left part of the figure) slide over the vertex producing a new one. It is a good exercise to try to visualize this sliding: it cannot be performed in $\mathbb{R}^{3}$; if we want the sliding region not to touch in its interior the other regions near the vertex, then it is necessary to use the fourth dimension. Hence we say that this move has 4-dimensional nature; it has the effect of modifying $P$ but not $M$. Note that even if the move "needs the fourth dimension", its result is still a locally flat polyhedron in $M$.

Of course, the above comments also apply to the inverses of the moves, that is, to the moves which in the figure go from right to left. Let us now clarify the meaning of the letters written on the regions of the polyhedra in the figure: they represent the gleams of the regions; note that only the $1 \rightarrow 2$ move changes the gleams of the regions near the vertex over which it is applied. Each move represents a modification of the embedded polyhedron, and hence produces a new polyhedron whose gleams (induced by the embedding in the ambient manifold as explained in Proposition 3.2) can differ from those of the initial polyhedron; moreover these gleams can change only on the regions which appear in the pictures, since otherwise the position of the regions in $M$ is left unchanged by the move. 
All these comments can be summarized in the following proposition:

Proposition 5.1. Let $P$ be a shadow of a 4-manifold $M$ and let $P^{\prime}$ be obtained from $P$ through the application of any sequence of $1 \rightarrow 2,0 \rightarrow 2$ and $2 \rightarrow 3$ embedded moves (and possibly their inverses). Then $P^{\prime}$ is a shadow of $M$. Moreover, the gleam of $P^{\prime}$ can be reconstructed from the gleam of $P$ by following step by step the changes in the gleams induced by the moves of the sequence.

The above moves change the polyhedron but do not change the ambient manifold; we now describe two moves which also change the manifold and which are useful in a number of applications we will outline in the next sections: the 0-bubble move and the \pm 1 -bubble move. On the level of naked polyhedra they are identical and shown in Figure 5; they differ on the level

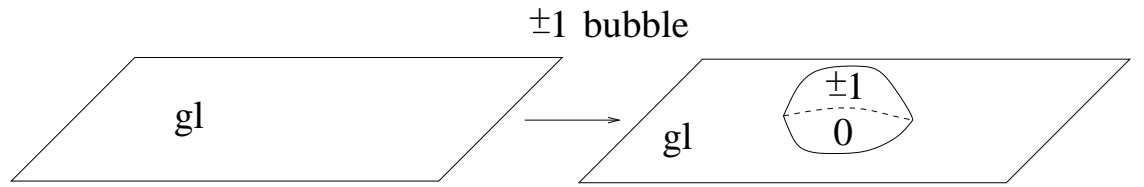

Fig. 5 . The \pm 1 -bubble moves: the gleam of the region over which the move is applied is left unchanged. A new 0 -gleam disc is created and a \pm 1 -gleam disc is attached to the boundary of the latter disc. The 0 -bubble move creates a 0 -gleam disc in place of the \pm 1 -gleam one.

of the gleams of the polyhedron after the move. The 0-bubble move applied to a polyhedron $P$ produces a polyhedron $P^{\prime}$ by attaching a 0 -gleam disc to a 0 -gleam disc contained in a region of $P$ and leaving unchanged the gleams of the other regions of the polyhedron; $P^{\prime}$ is not standard, but the construction of Theorem 4.1 can be generalized to simple polyhedra equipped with gleams. Let $M_{P}$ and $M_{P^{\prime}}$ be the 4-manifolds determined respectively by $P$ and $P^{\prime}$ as explained in Theorem 4.1; it can be checked that $M_{P^{\prime}}$ is diffeomorphic to the complement in $M_{P}$ of a properly embedded arc, that is, it is obtained by tunneling $M_{P}$. Conversely, $M_{P}$ can be recovered from $M_{P^{\prime}}$ by attaching a 3-handle whose cocore corresponds to that arc.

Let us now describe the effects of the \pm 1 -bubble moves. These moves are identical except the sign chosen; we concentrate on the 1-bubble move. Its application to a polyhedron $P$ produces a polyhedron $P^{\prime}$ with gleams by attaching a 1-gleam disc over a 0 -gleam disc in a region of $P$ and leaving the other gleams unchanged. It can be checked that if $M_{P}$ is the manifold determined by $P$ then the one determined by $P^{\prime}$ is $M_{P^{\prime}}=M_{P} \# \mathbb{C P}^{2}$, and in particular, $\partial M_{P}=\partial M_{P^{\prime}}$; analogously the application of a -1-bubble move produces a shadow of $M_{P} \# \overline{\mathbb{C P}}^{2}$. 
The last move we introduce is called the "trading" move and is found in Figure 6. Unlike the above moves, this move does not start from a con-
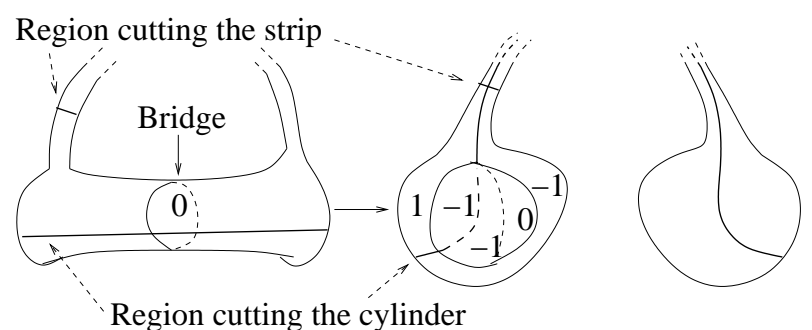

Fig. 6. In the left part of the figure we show the pattern to which the trading move applies. It consists of a cylinder cut by a 0-gleam disc and of a (possibly twisted) strip connecting the two ends of the cylinder. Some strands (corresponding to attaching curves of regions of the shadow) can pass over the cylinder or cut the strip. In the right part we show the effect of the trading move: the cylinder disappears, its two ends are capped off with two 0 -gleam discs and, in one of them, a little 0-gleam 2-disc is attached. The strands which before the move run across the cylinder, are now completed along the strip and linked with the little 0-gleam disc as shown in the drawing.

tractible sub-polyhedron of the initial polyhedron; hence we say that it is "non-local": one can apply it only when a pattern in the polyhedron which coincides with the left part of the figure is found. The move dramatically changes the topology of the polyhedron: in particular the fundamental group changes. For simplicity, in the figure we draw a version of the trading move in the case of a non-standard, simple polyhedron; up to the application of a suitable number of $0 \rightarrow 2$ moves, the polyhedron can be transformed into a standard one and the move can also be described in the standard setting but the drawings are more complicated and we omit them here.

Proposition $5.2([17])$. Let $P$ be an integer shadowed polyhedron and let $P^{\prime}$ be the polyhedron obtained by applying a trading move (or its inverse) to $P$. Then the manifold $M_{P^{\prime}}$ is obtained from $M_{P}$ by surgery along a closed curve $C$ embedded in the interior of $M_{P}$ (respectively, on an embedded sphere whose self-intersection number is zero), and then, in particular, $\partial M_{P}$ and $\partial M_{P^{\prime}}$ are diffeomorphic.

Proof. We limit ourselves to rough motivations for the above proposition; see [17] for details. We first analyze the portion of the manifold described in Figure 6 before the move. We define the bridge to be the part of the polyhedron $P$ formed by the curved cylinder together with the central disc with 0 -gleam in the left part of Figure 6 . It can be checked that there exists a handle decomposition of $M_{P}$ such that the bridge is contained in a 1-handle 
and the two ends of the bridge are contained in the two ends of the 1-handle. The other regions which in the figure run along the cylinder correspond to 2-handles whose attaching curves pass through the 1-handle. So, from the point of view of Kirby calculus, the left part of Figure 6 corresponds to the left part of Figure 7, where the strand running from one attaching sphere of the 1-handle to the other one corresponds to the attaching curve which in Figure 6 runs across the bridge.

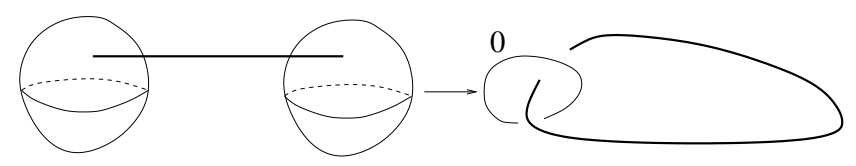

Fig. 7. In the left part of the figure, we show an example of a 1-handle in a Kirby calculus presentation of a 4-manifold over which an attaching curve of a 2-handle passes. In the right part we show the effect of trading the 1-handle for a 2-handle.

Now, let $C$ be a closed curve inside $M_{P}$ which passes once over the bridge in Figure 6 and then gets closed by the strip connecting the two ends of the cylinder; surgery along $C$ produces a 4-manifold which, in place of the 1handle determined by the bridge, contains a 2-handle whose attaching curve is an unknot in $\partial M_{P}$ and such that the attaching curve of every 2-handle which passed over the bridge, passes inside the disc bounded by the unknot (recall that surgery along a curve in a 4-manifold consists in replacing the regular neighborhood of the curve with a copy of $S^{2} \times D^{2}$ ).

It can be checked, but we will not do it here, that this move corresponds to the move which in Kirby calculus, given a presentation of a 3-manifold, allows one to exchange a 1-handle with a 0 -framed 2-handle represented by an unknot and linked with all the 2-handles passing over the 1-handle, obtaining a presentation of the same 3-manifold (see the right part of Figure 7).

We sum up the facts presented in this section in the following theorem. For a complete account we refer to [17].

TheORem 5.3. Let $P$ and $P^{\prime}$ be two integer shadowed polyhedra such that $P^{\prime}$ is obtained from $P$ by applying a move of the following types:

(1) $2 \rightarrow 3$ move or its inverse;

(2) finger move or its inverse;

(3) $1 \rightarrow 2$ move or its inverse.

Then $M_{P}$ and $M_{P^{\prime}}$ are diffeomorphic by an orientation preserving diffeomorphism.

If $P^{\prime}$ is obtained from $P$ by a \pm 1 -bubble move then $M_{P^{\prime}}$ is diffeomorphic to the boundary connected sum of $M_{P}$ and a punctured $\overline{\mathbb{C P}^{2}}$ or $\mathbb{C P}^{2}$ 
respectively, hence $\partial M_{P^{\prime}}$ is diffeomorphic (by an orientation preserving diffeomorphism) to $\partial M_{P}$. If $P^{\prime}$ is obtained from $P$ by a 0-bubble move then $M_{P^{\prime}}$ is diffeomorphic to the boundary connected sum of $M_{P}$ and $S^{2} \times D^{2}$, and then in particular $\partial M_{P^{\prime}}=\partial M_{P} \# S^{2} \times S^{1}$. Finally, if $P^{\prime}$ is obtained from $P$ by the application of a trading move, then $M_{P^{\prime}}$ is obtained from $M_{P}$ by surgery along the curve which passes once over the bridge and once over the cylinder of Figure 6 ; in particular $\partial M_{P^{\prime}}=\partial M_{P}$.

6. Some applications and questions. In this section we briefly recall some results and open questions. The first one emerges once one proves Theorem 4.1 and is the following:

Question 6.1. Given two shadows $P$ and $P^{\prime}$ of the same manifold, can they be connected by a sequence of suitable moves, for instance shadow equivalences (see the beginning of Section 5)?

A first answer to the above question is due to Turaev [17]:

Theorem 6.2. Let $P$ and $P^{\prime}$ be two shadows of the same 4-manifold $M$. Then they can be connected by a sequence of shadow equivalences, 0-bubble moves and their inverses. Moreover the sequence always contains the same number of 0-bubble moves and of inverse 0-bubble moves.

In the above result there is a disturbing aspect: the sequence of moves connecting $P$ and $P^{\prime}$ can contain 0-bubble moves and can produce at a certain step a polyhedron which is not a shadow of $M$, hence in general the sequence does not pass only through shadows of $M$. Moreover the converse of the above theorem is not yet proved:

QUESTION 6.3. Let $P$ and $P^{\prime}$ be obtained from each other by a sequence of shadow equivalences and 0-bubble moves containing the same number of 0-bubble moves and of their inverses. Is it true that the manifold $M$ and $M^{\prime}$ determined by $P$ and $P^{\prime}$ respectively are diffeomorphic?

The author proved in [4] that the answer to the above question is "yes" when $P$ (and hence $P^{\prime}$ ) is simply connected; the positive answer in general is still a conjecture.

The following question can be thought of as an embedded version of the Andrews-Curtis conjecture:

Question 6.4. Let $P$ and $P^{\prime}$ be shadows of the same manifold; can one connect them by a sequence of shadow equivalences? (No bubble moves!)

By Matveev and Piergallini's results ([13], [14]), the analogous question in dimension 3 has a positive answer. The 4-dimensional case remains open; it has to be pointed out here that, although apparently the above question 
is strictly related to the Andrews-Curtis conjecture, until now no formal relation between the two problems has been proved.

Theorem 6.2 can be rewritten in terms of tunnel shadows of closed 4manifolds, and it then states that any two shadows of a closed 4-manifold are connected by a sequence of shadow equivalences and 0-bubble moves. In this case, it represents a genuine calculus in the standard sense.

7. Shadows of 3-manifolds. In this section we will briefly recall the notion of a shadow of a 3-manifold and give an overview of some recent results on the subject.

Definition 7.1. Let $(P, \mathrm{gl})$ be a shadow of a 4-manifold $M$; then $(P, \mathrm{gl})$ is a shadow of the 3-manifold $N=\partial M$.

It can be proved that any closed and oriented 3-manifold admits a shadow, and even a simply connected one; moreover, the application of any shadow equivalence or \pm 1 -bubble move to a shadow of a 3 -manifold produces a shadow of the same manifold. In general, the following was proved in $[8]$ :

Theorem 7.2 (Calculus for shadows of 3-manifolds). Any two simply connected shadows of the same closed and oriented 3-manifold can be connected by a sequence of shadow equivalences, \pm 1 -bubble moves and their inverses.

The above result is an analogue of the Kirby calculus in the world of shadows of 3-manifolds, where the blow-up move is replaced by the \pm 1 bubble moves; its pretty feature is that it is based only on local moves.

A new notion of complexity of 3-manifolds based on shadows is defined in $[9]$ :

Definition 7.3. Let $N$ be a closed and oriented 3-manifold. The shadow complexity of $N$ is the minimal number of vertices of a (possibly nonstandard) shadow of $N$.

EXAMPLE 7.4. As shown in Example 4.7, the polyhedron $S^{2}$ equipped with gleam -1 is a shadow of $S^{3}$ and hence the shadow complexity of $S^{3}$ is zero.

Strong relations have been found between shadow complexity and hyperbolic geometry of 3-manifolds. The following theorem, to appear in [9], summarizes some of them:

THEOREM 7.5. There exist universal positive constants $h_{1}$ and $h_{2}$ such that for any hyperbolic, complete 3-manifold $N$ with finite volume,

$$
h_{1} \operatorname{Vol}(N) \leq \operatorname{sc}(N) \leq h_{2}(\operatorname{Vol}(N))^{2},
$$


where $\operatorname{sc}(N)$ is the shadow complexity of $N$ and $\operatorname{Vol}(N)$ is its hyperbolic volume.

Moreover, in the same paper we prove that each 3-dimensional graph manifold admits a shadow containing no vertices.

These results suggest that a study of hyperbolic manifolds by means of shadows can be attempted; to that end, one of the first questions to answer is the following:

QUESTION 7.6. Given a polyhedron with gleams $(P, \mathrm{gl})$, what are the necessary and sufficient conditions on $(P, \mathrm{gl})$ ensuring that the 3 -manifold determined by the shadow is irreducible, or atoroidal, or hyperbolic?

A clear and definitive combinatorial condition answering the above question is still missing. Partial answers have been obtained in [7]: for instance, it has been proved that if a 3 -manifold admits a standard shadow whose gleams are all greater than 7 in absolute value, then the manifold admits a metric with negative sectional curvature, and consequently, is aspherical and atoroidal.

8. A glance on branched shadows. Besides the open problems presented in the preceding sections, which, in part, are inherent to the theory of shadows, a study of additional structures and invariants of 4-manifolds can be attempted by means of shadows. In this section we summarize the results proved by the author in [4] (and which will be presented in a self-contained way in [5] and [6]) in this direction and based on the notion of branched shadow.

Given a simple polyhedron $P$ we define the notion of branching on it as follows:

Definition 8.1. A branching $b$ on $P$ is a choice of an orientation for each region of $P$ such that for each edge of $\operatorname{Sing}(P)$, the orientations induced on the edge by the regions containing it do not coincide.

Not all the simple polyhedra admit a branching and, on the other hand, there are some which admit many different ones. So, we will say that a polyhedron is branchable if it admits a branching and we define a branched polyhedron, a pair $(P, b)$ where $b$ is a branching on the polyhedron $P$.

Definition 8.2. Let $(P, \mathrm{gl})$ be a shadow of an oriented 4-manifold $M ; P$ is said to be branchable if the underlying polyhedron is. A branched shadow of $M$ is a triple $(P, \mathrm{gl}, b)$ where $(P, \mathrm{gl})$ is a shadow and $b$ is a branching on the underlying polyhedron. When this does not cause any confusion, we do not specify the branching $b$ nor the gleam gl and we will simply write $P$. 

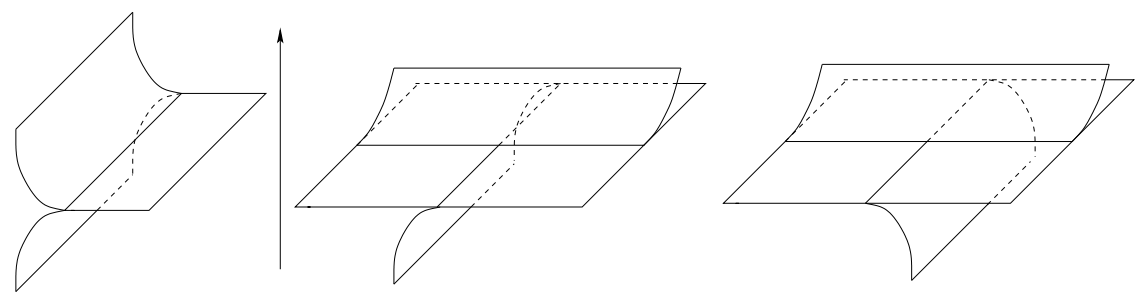

Fig. 8. How a branching allows a smoothing of the polyhedron

A branching on a simple polyhedron allows us to smoothen its singularities and equip it with a smooth structure as shown in Figure 8.

Using the orientation and smooth structure of the shadow, in [5] (see also [4]), we show how to equip the regular neighborhood of a shadow in a 4manifold with a pair of mutually transverse distributions of oriented 2-planes (roughly speaking, the planes which in Figure 8 are horizontal and their orthogonal complements). In the same paper, using this pair of distributions of oriented 2-planes, we show how to fix a homotopy class of almost complex structures on the regular neighborhood of the shadow, and hence a Spin ${ }^{c}$ structure on this manifold. The result in [5] can be summarized as follows:

THEOREM 8.3. Let $M$ be a 4-manifold admitting a shadow and let $s$ be a $\mathrm{Spin}^{c}$-structure on $M$. There exists a branched shadow $P$ of $M$ representing s. Stated differently, given a homotopy class $[J]$ of almost complex structures on $M$, there exists a branched shadow of $M$ representing $[J]$.

Following the last part of Theorem 8.3, one can study how branched shadows can be used to find genuine complex structures on their thickening. In [5], the problem is attacked through techniques that are similar to those already used for real surfaces in complex manifolds by Bishop in [2] and Harlamov-Eliashberg in [11], based on counting the number of complex elliptic and hyperbolic points. The result we obtain can be summarized as follows:

THEOREM 8.4 ([5]). Let $P$ be a branched shadow reconstructing the manifold $M$ equipped with the almost complex structure $J$. There exists a genuine complex structure $J^{\prime}$ homotopic to $J$ such that $P$ contains no $J^{\prime}$-complex points of negative type, that is, $P$ contains no points where its tangent plane is $J^{\prime}$-complex but oriented differently by the branching on $P$ and its complex structure induced by $J^{\prime}$.

As a natural development of the above result, using Gompf-Eliashberg's constructions, combinatorial sufficient conditions on a branched shadow ensuring that its thickening admits a Stein domain structure will be studied in [6] (see also [4, Chapter V] ). The conditions we find are based on the definition (which we will not recall here), for each region of a branched 
polyhedron, of an integer coefficient called the Euler number which is easily calculable from the combinatorial structure of the polyhedron. The main result, which will be presented in a self-contained way in [6], is summarized in a simplified version in the following:

TheOREM 8.5 ([6]). Let $P$ be a branched shadow of a 4-manifold $M$. There exist constants $k_{i}$ such that if $\mathrm{Eul}_{i}+\mathrm{gl}_{i} \leq k_{i}$ for each region $R_{i}$ of $P$, where $\mathrm{gl}_{i}$ is the gleam of $R_{i}$ and $\mathrm{Eul}_{i}$ is its Euler number, then $M$ admits a Stein structure whose underlying complex structure belongs to the homotopy class of almost complex structures determined by $P$.

The conditions found in the above theorem have been exploited in [4] to explicitly exhibit minimal genus representatives of some particular homology classes of surfaces in 4-manifolds:

COROLlary 8.6. Let $P$ be a branched shadow satisfying the hypotheses of Theorem 8.4 and such that $\mathrm{gl}_{i} \geq 0$ for every $i$, and let $[S] \in H_{2}(P ; \mathbb{Z})$ be a homology class of the form $\sum_{i} n_{i} R_{i}$ where $R_{i}$ are the regions of $P$ and $n_{i}$ are non-negative integers. Then there is an explicit construction of an oriented embedded representative $S^{\prime} \subset M$ of $[S]$ having the lowest possible genus in its homology class.

Moreover, using Theorem 8.4 on branched shadows equipped with 0gleams on every region, the following result, which was conjectured by Benedetti and Petronio in [1], is proved:

THEOREM 8.7. Let $P$ be a branched spine of an oriented 3-manifold $N$ such that the Euler number of each region of $P$ is non-positive. Then the homotopy class of oriented 2-planes encoded by the branching of the spine on its 3-dimensional thickening contains a representative distribution which is a tight contact structure.

The above results are likely to be non-optimal and a further study is required to fully understand the potential of the theory of branched shadows.

\section{References}

[1] R. Benedetti and C. Petronio, Branched spines and contact structures on 3-manifolds, Ann. Mat. Pura Appl. (4) 178 (2000), 81-102.

[2] E. Bishop, Differentiable manifolds in complex euclidean space, Duke Math. J. 32 (1975), 1-21.

[3] U. Burri, For a fixed Turaev shadow Jones-Vassiliev invariants depend polynomially on the gleams, Comment. Math. Helv. 72 (1997), 110-127.

[4] F. Costantino, Shadows and branched shadows of 3- and 4-manifolds, Tesi di Perfezionamento, Scuola Normale Superiore, Pisa, 2004.

[5] - Branched shadows and complex structures of 4-manifolds, xxx.arXiv.org/math. GT/0502292. 
[6] F. Costantino, Stein domains and branched shadows of 4-manifolds, xxx.arXiv.org/ math.CV/0504387 (2005).

[7] F. Costantino, R. Frigerio, B. Martelli and C. Petronio, Triangulations of 3-manifolds, hyperbolic relative handlebodies, and Dehn filling, xxx.arXiv.org/math.GT/ 0402339 (2004).

[8] F. Costantino and D. P. Thurston, A shadow calculus for 3-manifolds, xxx.arXiv. org/math.GT/0506577.

[9] - - - 3-manifolds efficiently bound 4-manifolds, in preparation.

[10] M. N. Goussarov, Interdependent modifications of links and invariants of finite degree, Topology 37 (1998), 595-602.

[11] V. M. Harlamov and Y. Eliashberg, On the number of complex points of a real surface in a complex surface, in: Proc. Leningrad Int. Topology Conf., 1982, $143-148$.

[12] F. Laudenbach and V. Poenaru, A note on 4-dimensional handlebodies, Bull. Soc. Math. France 100 (1972), 337-344.

[13] S. Matveev, Transformations of special spines and the Zeeman conjecture, Math. USSR-Izv. 31 (1988), 423-434.

[14] R. Piergallini, Standard moves for standard polyhedra and spines, Rend. Circ. Mat. Palermo 37 (1988), suppl. 18, 391-414.

[15] A. Shumakovitch, Shadow formula for the Vassiliev invariant of degree two, Topology 36 (1997), 449-469.

[16] D. P. Thurston, The algebra of knotted trivalent graphs and Turaev's shadow world, Geom. Topol. Monogr. 4 (2002), 337-362.

[17] V. G. Turaev, Quantum Invariants of Knots and 3-Manifolds, de Gruyter Stud. Math. 18, de Gruyter, Berlin, 1994.

[18] -, Quantum invariants of 3-manifolds and a glimpse of shadow topology, C. R. Acad. Sci. Paris Sér. I Math. 313 (1991), 395-398.

[19] —, Shadow links and face models of statistical mechanics, J. Differential Geom. 36 (1992), 35-74.

Institut de Recherche Mathématique Avancée

Rue René Descartes 7

67084 Strasbourg, France

E-mail: f.costantino@sns.it

Received 5 November 2004;

in revised form 22 January 2005 